\title{
A FINITE-DIMENSIONAL INTEGRABLE SYSTEM ASSOCIATED WITH A POLYNOMIAL EIGENVALUE PROBLEM
}

TAIXI XU, WEIHUA MU, AND ZHIJUN QIAO

Received 6 February 2006; Accepted 21 March 2006

M. Antonowicz and A. P. Fordy (1988) introduced the second-order polynomial eigenvalue problem $L \phi=\left(\partial^{2}+\sum_{i=1}^{n} v_{i} \lambda^{i}\right) \phi=\alpha \phi(\partial=\partial / \partial x, \alpha=$ constant $)$ and discussed its multi-Hamiltonian structures. For $n=1$ and $n=2$, the associated finite-dimensional integrable Hamiltonian systems (FDIHS) have been discussed by Xu and Mu (1990) using the nonlinearization method and Bargmann constraints. In this paper, we consider the general case, that is, $n$ is arbitrary, provide the constrained Hamiltonian systems associated with the above-mentioned second-order polynomial ergenvalue problem, and prove them to be completely integrable.

Copyright (c) 2006 Hindawi Publishing Corporation. All rights reserved.

\section{Introduction}

In classical mechanics one describes the equation of motion by Hamiltonian systems of the form [2]

$$
\frac{d q_{j}}{d t}=\frac{\partial H}{\partial p_{j}} \quad \frac{d p_{j}}{d t}=-\frac{\partial H}{\partial q_{j}} \quad(j=1, \ldots, n),
$$

where $q=\left(q_{1}, \ldots, q_{n}\right) \in \mathbb{R}^{n}, p=\left(p_{1}, \ldots, p_{n}\right) \in \mathbb{R}^{n}$, and $H=H(q, p)$ is a smooth function on an open domain $\Omega$ of $\mathbb{R}^{2 n}$.

It is customary to introduce the "Poisson bracket" $\{F, G\}$ for two functions $F, G \in$ $C^{1}(\Omega)$ by [7]

$$
\{F, G\}=\sum_{j=1}^{n}\left(\frac{\partial F}{\partial q_{j}} \frac{\partial G}{\partial p_{j}}-\frac{\partial F}{\partial p_{j}} \frac{\partial G}{\partial q_{j}}\right)=\left\langle F_{q}, G_{p}\right\rangle-\left\langle F_{p}, G_{q}\right\rangle .
$$

$F, G$ are called in involution if $\{F, G\}=0$. 
Using a notation borrowed from differential geometry we associate with (1.1) the "vector field" or first-order differential operator

$$
X_{H}=\sum_{j=1}^{n}\left(H_{p_{j}} \frac{\partial}{\partial q_{j}}-H_{q_{j}} \frac{\partial}{\partial p_{j}}\right) .
$$

Definition 1.1. A nonconstant function $F \in C^{\prime}(\Omega)$ is called an integral of $X_{H}$ if

$$
X_{H} F=\{F, H\}=0 \text {. }
$$

Definition 1.2. A Hamiltonian vector field $X_{H}$ in $\Omega \subset \mathbb{R}^{2 n}$ is called "integrable" if it possesses $n$ integrals $F_{j} \in C^{1}(\Omega)$ satisfying the following conditions

(i) $\left\{F_{j}, H\right\}=0$,

(ii) $\left\{F_{j}, F_{k}\right\}=0$,

(iii) the gradients $d F_{j}$ are linearly independent in $\Omega$.

The first condition expresses that the $F_{j}$ are integrals; the second one means that any two such integrals commute. The third condition is a requirement for nondegeneracy, which we will have to relax frequently.

Since Cao introduced the nonlinearization method to search for finite-dimensional completely integrable Hamiltonian systems [3-6] associated with soliton equations, numerous such systems have been obtained by many mathematicians $[8-12,14,15]$.

In this paper, we consider the integrable systems associated with the polynomial eigenvalue problem

$$
L \phi=\left(\partial^{2}+\sum_{i=1}^{n} v_{i} \lambda^{i}\right) \phi=\alpha \phi(\partial=\partial / \partial x, \alpha=\text { constant })
$$

When $n=1$, denote $v_{1}$ by $v$, (1.5) becomes

$$
\phi_{x x}+\lambda v \phi=\alpha \phi
$$

Equation (1.6) is associated with the Harry-Dym (HD) equation

$$
v_{t}=\left(\frac{1}{2 \sqrt{v}}\right)_{x x x}+\left(\frac{2 \alpha}{\sqrt{v}}\right)_{x} .
$$

Remark 1.3. Actually, spectral problem (1.6) generates the Camassa-Holm $(\mathrm{CH})$ equation in its negative-order hierarchy, whereas it produces the HD equation (1.7) in its positiveorder hierarchy [9]. Both hierarchies are integrable. When $n=2, v_{1}=u, v_{2}=v,(1.5)$ becomes

$$
\phi_{x x}+\left(\lambda u+\lambda^{2} v\right) \phi=\alpha \phi
$$


Equation (1.8) is associated with the following coupled Harry-Dym (CHD) equation:

$$
\begin{gathered}
u_{t}=\left(\frac{1}{2 \sqrt{v}}\right)_{x x x}-2 \alpha\left(\frac{1}{\sqrt{v}}\right)_{x}, \\
v_{t}=2 u \frac{1}{\sqrt{v}}+u_{x} \frac{1}{\sqrt{v}} .
\end{gathered}
$$

We have already obtained the integrable Hamiltonian systems associated with HarryDym and coupled Harry-Dym equations [13]. For general positive integer $n$, the associated integrable system is given in this paper. In the next section, we give the Hamiltonian system associated with the polynomial eigenvalue problem, and in Section 3, we obtain the involutive integrals and prove they are linearly independent.

\section{The Hamiltonian system}

Consider the evolution equation

$$
\phi_{t_{m}}=-\frac{1}{2} B_{x}^{(m)} \phi+B^{(m)} \phi_{x}
$$

where

$$
B^{(m)}=\sum_{j=0}^{m-1} b_{j} \lambda^{m-j}, \quad b_{-j}=0(j=1,2, \ldots) .
$$

From the solvability condition of (1.5) and (2.1), the hierarchy of evolution equations of potentials $v=\left(v_{1}, \ldots, v_{n}\right)^{T}$ can be written as

$$
v_{i}=\left[\begin{array}{cccc}
0 & \cdots & 0 & J_{0} \\
\vdots & \cdot & \cdot & J_{1} \\
0 & \cdot & \cdot & \vdots \\
J_{0} & J_{1} & \cdots & J_{n-1}
\end{array}\right]\left[\begin{array}{c}
b_{m-n} \\
b_{m-n-1} \\
\vdots \\
b_{m-1}
\end{array}\right]
$$

Also, from the solvability condition, it is found that $b_{k}$ satisfies

$$
J_{0} b_{j}+J_{1} b_{j+1}+\cdots+J_{n} b_{j+n}=0
$$

or

$$
K\left(b_{j}, b_{j+1}, \ldots, b_{j+n-1}\right)^{T}=J\left(b_{j+1}, b_{j+2}, \ldots, b_{j+n}\right)^{T},
$$

or

$$
K G_{j}=J G_{j+1},
$$


4 A finite-dimensional integrable system

where

$$
K=\left[\begin{array}{cccc}
0 & \cdots & 0 & J_{0} \\
\vdots & . & . & J_{1} \\
0 & . & . & \vdots \\
J_{0} & J_{1} & \cdots & J_{n-1}
\end{array}\right], \quad J=\left[\begin{array}{ccccc}
0 & \cdots & 0 & J_{0} & 0 \\
\vdots & . & . & J_{1} & 0 \\
0 & \cdot & . & \vdots & \vdots \\
J_{0} & J_{1} & \cdots & J_{n-2} & 0 \\
0 & 0 & \cdots & 0 & -J_{n}
\end{array}\right]
$$

are the Lenard pair of operators

$$
\begin{gathered}
G_{j}=\left(b_{j}, b_{j+1}, \ldots, b_{j+n-1}\right), \\
J_{0}=\frac{1}{2} \partial^{3}-2 \alpha \partial, \quad J_{i}=v_{i} \partial+\partial v_{i} \quad(i=1,2, \ldots, n) .
\end{gathered}
$$

It is evident that if $\phi$ is a solution of (1.5), then

$$
\sum_{i=0}^{n} \lambda^{i} J_{i} \phi^{2}=0
$$

Rewrite it as

$$
\sum_{i=0}^{n} \lambda^{i} J_{i} P=0
$$

Letting $P=\sum_{j=0}^{\infty} P_{j} \lambda^{-j}$; we find that $P_{j}$ satisfies the same relationship (2.4) as $b_{j}$ does. Multiplying both sides of (2.10) by $P$ and integrating it once, we get

$$
P_{x x} P-\frac{1}{2} P_{x}^{2}-2 \alpha P^{2}+2 \sum_{i=1}^{n} \lambda^{i} v_{i} P^{2}=C(\lambda) .
$$

Set $P_{0}=V_{n}^{-1 / 2}, C(\lambda)=\lambda^{n}$. By substituting $P=\sum_{j=0}^{\infty} P_{j} \lambda^{-j}$ into (2.11), we find that $P_{j}=$ $b_{j}$,

$$
\begin{aligned}
b_{k+n} b_{0}^{-1}= & -\frac{1}{4} \sum_{j=0}^{k} b_{j x x} b_{k-j}+\frac{1}{8} \sum_{j=0}^{k} b_{j x} b_{k-j, x}+\frac{\alpha}{2} \sum_{j=0}^{k} b_{j} b_{k-j} \\
& -\frac{1}{2} \sum_{i=0}^{n-1} \sum_{j=0}^{k+i} b_{j} b_{k+i-j}-\frac{1}{2} v_{n} \sum_{j=1}^{k+n-1} b_{j} b_{k+n-j} \quad(k=1,2, \ldots) .
\end{aligned}
$$

Proposition 2.1. Let $\lambda_{j}$ be an eigenvalue of (1.5) and $\phi_{j}$ an eigenfunction corresponding to $\lambda_{j}$. Then

$$
\begin{gathered}
\operatorname{grad} \lambda_{j}=\left(\frac{\delta \lambda_{j}}{\delta v_{1}}, \ldots, \frac{\delta \lambda_{j}}{\delta \nu_{1}}\right)^{T}=\left(\lambda_{j} \phi_{j}^{2}, \lambda_{j}^{2} \phi_{j}^{2}, \ldots, \lambda_{j}^{n} \phi_{j}^{2}\right)^{T}, \\
K \operatorname{grad} \lambda_{j}=\lambda_{j} J \operatorname{grad} \lambda_{j} .
\end{gathered}
$$


Define the Lenard sequence recursively: $G_{0}=\left(b_{0}, \ldots, b_{n-1}\right)^{T}, K G_{j-1}=J G_{j}(j=1$, $2, \ldots), X_{j}=J G_{j}(j=0,1,2, \ldots)$ are the CHD vector fields.

Let

$$
G_{0}=\sum_{j=1}^{N} \operatorname{grad} \lambda_{j}
$$

Then

$$
b_{j}=\left\langle\Lambda^{j+1} \phi, \phi\right\rangle \quad(j=0,1,2, \ldots, n-1),
$$

where $\langle\cdot, \cdot\rangle$ is the standard inner-product in $\mathbb{R}^{N}, \Lambda=\operatorname{diag}\left(\lambda_{1}, \ldots, \lambda_{N}\right)$.

From (2.4), (2.14), and (2.15), we have

$$
\sum_{j=0}^{k-1} J_{n-j}\left\langle\Lambda^{k-j} \phi, \phi\right\rangle=0 \quad(k=1,2, \ldots, n),
$$

which yields

$$
v_{n-k}=\langle\Lambda \phi, \phi\rangle^{-1} \partial^{-1}\langle\Lambda \phi, \phi\rangle\left[J_{n}\left\langle\Lambda^{k} \phi, \phi\right\rangle+\cdots+J_{n-k+2}\left\langle\Lambda^{2} \phi, \phi\right\rangle\right] .
$$

By making use of the recursion formula of $v_{k}$, we have the following proposition.

Proposition 2.2. The constraint between the potentials and the eigenfunctions (1.5) is of the form

$$
\begin{gathered}
v_{n}=\langle\Lambda \phi, \phi\rangle^{-2}, \\
v_{n-k}=\sum_{j=1}^{k} a_{j}\langle\Lambda \phi, \phi\rangle^{-(j+2)} \sum_{l_{1}+\cdots+l_{j}=k-j}\left\langle\Lambda^{l_{1}+2} \phi, \phi\right\rangle \cdots\left\langle\Lambda^{l_{j}+2} \phi, \phi\right\rangle \quad(k=1,2, \ldots, n-1),
\end{gathered}
$$

where $a_{j}=(-1)^{j}(j+1), a_{0}=1$, and $\phi_{j}, \lambda_{j}$ satisfy (1.5).

We now consider the following system instead of (1.5):

$$
\phi_{j x x}+\sum_{i=1}^{n} v_{i} \lambda_{j}^{i} \phi_{j}=\alpha \phi_{j} \quad(j=1,2, \ldots, N),
$$

where $\lambda_{j} \neq \lambda_{k}$ when $j \neq k$. Let

$$
q=\left(q_{1}, q_{2}, \ldots, q_{N}\right)^{T}=\left(\phi_{1}, \phi_{2}, \ldots, \phi_{N}\right)^{T}
$$

then (2.20) can be condensed as

$$
q_{x x}+\sum_{i=1}^{n} v_{i} \Lambda^{i} q=\alpha q .
$$


6 A finite-dimensional integrable system

By using the identity

$$
\sum_{i=1}^{k}\left\langle\Lambda^{k-j} q, q\right\rangle \sum_{j=1}^{i} a_{j} \gamma_{i, j}=\sum_{i=1}^{k} a_{j} \sum_{i=0}^{k-j}\left\langle\Lambda^{i} q, q\right\rangle \gamma_{k-i, j}
$$

and substituting (2.18), (2.19) into (2.22), we get

$$
p_{x}=\alpha q-\sum_{i=0}^{n-1} a_{j}\langle\Lambda q, q\rangle^{i+2} \cdot \sum_{\substack{l_{1}+\cdots+l_{i+1} \\ q_{x}=p}}\left\langle\Lambda^{l_{1}+2} q, q\right\rangle \cdots\left\langle\Lambda^{l_{i+2}} q, q\right\rangle \Lambda^{l_{i+1}+2} q,
$$

which can be written in canonical Hamiltonian system

$$
q_{x}=\frac{\partial H_{0}}{\partial p}, \quad p_{x}=-\frac{\partial H_{0}}{\partial q},
$$

where $p=\left(p_{1}, \ldots, p_{N}\right)^{T}=\left(q_{1 x}, \ldots, q_{N x}\right)^{T}$,

$$
\begin{gathered}
H_{0}=\frac{1}{2}\langle p, p\rangle-\frac{\alpha}{2}\langle q, q\rangle+\frac{1}{2} \sum_{i=0}^{n-2} \bar{b}_{i}\langle\Lambda q, q\rangle^{-(i+2)} \cdot \sum_{l_{1}+\cdots+l_{i+1}=n-2-i}\left\langle\Lambda^{l_{1}+2} q, q\right\rangle \cdots\left\langle\Lambda^{l_{i+1}+2} q, q\right\rangle \\
\bar{b}_{i}=\frac{a_{i}}{i+1}=(-1)^{i}, i=0,1, \ldots
\end{gathered}
$$

\section{Involutivity and integrability}

Consider the constraint of (2.12)

$$
\begin{aligned}
F_{n+k}= & \frac{b_{k+n}}{b_{0}}+\frac{1}{4} \sum_{j=0}^{k} b_{j x x} b^{k-j}-\frac{1}{8} \sum_{j=0}^{k} b_{j x} b_{k-j, x} \\
& \left.+\frac{1}{2} \sum_{i=1}^{n-1} v_{i} \sum_{j=0}^{k+i} b_{j} b_{k+i-j}-\frac{\alpha}{2} \sum_{j=0}^{k} b_{j}^{k-j}+\frac{1}{2} v_{n} \sum_{j=1}^{k+n-1} b_{j} b_{k+n-j}\right]\left.\right|_{A},
\end{aligned}
$$

where subscript $A$ means to substitute $b_{j}=\left\langle\Lambda^{j+1} q, q\right\rangle$ into (2.12). So

$$
\begin{aligned}
F_{n+k}= & \frac{1}{2} \sum_{j=0}^{k-1}\left\langle\Lambda^{j+1} q, p_{x}\right\rangle\left\langle\Lambda^{k-j-1} q, q\right\rangle+\frac{1}{2} \sum_{j=0}^{k}\left\langle\Lambda^{j+1} p, p\right\rangle\left\langle\Lambda^{k-j+1} q, q\right\rangle \\
& +\frac{1}{2}\left\langle\Lambda^{k+1} q, p_{x}\right\rangle\langle\Lambda q, q\rangle-\frac{1}{2}\left\langle\Lambda^{j+1} q, p\right\rangle\left\langle\Lambda^{k-j+1} p, q\right\rangle \\
& -\frac{\alpha}{2} \sum_{j=0}^{k}\left\langle\Lambda^{j+1} q, q\right\rangle\left\langle\Lambda^{k-j+1} q, q\right\rangle+\frac{1}{2} \sum_{i=0}^{n-1} v_{i} \sum_{j=0}^{k+i}\left\langle\Lambda^{j+1} q, q\right\rangle\left\langle\Lambda^{k+i-j+1} q, q\right\rangle \\
& +\frac{1}{2} v_{n} \sum_{j=1}^{k+n-1}\left\langle\Lambda^{j+1} q, p\right\rangle\left\langle\Lambda^{k+i-j+1} q, q\right\rangle+\langle\Lambda q, q\rangle^{-1}\left\langle\Lambda^{k+n+1} q, q\right\rangle .
\end{aligned}
$$


Through direct calculations from (2.18), (2.19), and (2.24), we have

$$
\begin{aligned}
F_{n+k}= & \frac{1}{2} \sum_{i=0}^{n-1} \bar{b}_{i}\langle\Lambda q, q\rangle^{-(i+1)} \cdot \sum_{l_{1}+\cdots+l_{i+1}=n-1-i}\left\langle\Lambda^{l_{1}+2} q, q\right\rangle \cdots\left\langle\Lambda^{l_{i}+2} q, q\right\rangle\left\langle\Lambda^{i_{i+1}+k+2} q, q\right\rangle \\
& +\frac{1}{2} \sum_{j=0}^{k}\left[\left\langle\Lambda^{j+1} p, p\right\rangle\left\langle\Lambda^{k-j+1} q, q\right\rangle-\left\langle\Lambda^{j+1} q, q\right\rangle\left\langle\Lambda^{k-j+1} p, q\right\rangle\right], \quad k=1,2, \ldots
\end{aligned}
$$

Set

$$
\begin{gathered}
G_{k}=\frac{1}{2} \sum_{j=0}^{k}\left[\left\langle\Lambda^{j+1} p, p\right\rangle\left\langle\Lambda^{j+1} q, q\right\rangle-\left\langle\Lambda^{j+1} q, p\right\rangle\left\langle\Lambda^{k-j+1} p, q\right\rangle\right], \\
Q_{k}=F_{k+n}-G_{k} .
\end{gathered}
$$

It is known (see Cao [3]) that $G_{k}$ are in involution. Using the identity

$$
\begin{aligned}
\sum_{i=0}^{l}\left\langle\Lambda^{l+k+j-i} p, p\right\rangle\left\langle\Lambda^{i} q, q\right\rangle+\sum_{i=0}^{k}\left\langle\Lambda^{i} p, p\right\rangle\left\langle\Lambda^{l+k+j-i} q, q\right\rangle \\
=\sum_{i=0}^{l+k+j}\left\langle\Lambda^{l+k+j-i} p, p\right\rangle\left\langle\Lambda^{i} q, q\right\rangle-\sum_{i=l+1}^{l+j-1}\left\langle\Lambda^{l+k+j-i} p, p\right\rangle\left\langle\Lambda^{i} q, q\right\rangle,
\end{aligned}
$$

we can show by straightforward calculations that

$$
\left\{Q_{k}, G_{l}\right\}+\left\{G_{k}, Q_{l}\right\}+\left\{Q_{k}, Q_{l}\right\}=0 \text {. }
$$

So

$$
\left\{F_{k+n}, F_{l+n}\right\}=0 \text {. }
$$

Since all $\lambda_{k}$ are distinct, the Vandermonde determinant of $\lambda_{1}, \lambda_{2}, \ldots, \lambda_{N}$ is not zero. Then it is easy to see that

$$
\operatorname{grad} F_{n+k}=\left(\frac{\partial F_{n+k}}{\partial q_{1}}, \ldots, \frac{\partial F_{n+k}}{\partial q_{N}}, \frac{\partial F_{n+k}}{\partial p_{1}}, \ldots, \frac{\partial F_{n+k}}{\partial p_{N}}\right), \quad k=1,2, \ldots
$$

are functionally independent. So we have the following proposition.

Proposition 3.1. The Hamiltonian system $\left(\mathbb{R}^{2 N}, d p \wedge d q, H_{0}\right)$ is completely integrable in the sense of Liouville.

Consider the systems obtained from (2.1)

$$
\phi_{j t_{m}}=-\frac{1}{2} R_{x}^{(m)} \phi_{j}+R^{(m)} \phi_{j x} \quad(j=1,2, \ldots, N) .
$$


Substituting $b-j=\left\langle\Lambda^{j+1} q, q\right\rangle(j=1,2, \ldots)$ into it, we have

$$
\begin{aligned}
q_{t_{m}}= & \sum_{j=0}^{m-1}\left[\left\langle\Lambda^{j+1} q, q\right\rangle \Lambda^{m-j} p-\left\langle\Lambda^{j+1} q, p\right\rangle \Lambda^{m-j} q\right], \\
p_{t_{m}}= & \frac{\partial}{\partial x} q_{t_{m}}=\sum_{j=0}^{m-1}\left[\left\langle\Lambda^{j+1} q, p\right\rangle \Lambda^{m-j} p-\left\langle\Lambda^{j+1} p, p\right\rangle \Lambda^{m-j} q\right] \\
& +\sum_{j=0}^{m-1}\left[\left\langle\Lambda^{j+1} q, q\right\rangle \Lambda^{m-j} p_{x}-\left\langle\Lambda^{j+1} q, p_{x}\right\rangle \Lambda^{m-j} q\right] .
\end{aligned}
$$

Through direct calculation from (2.24), (3.10) can be written in canonical Hamiltonian system

$$
q_{t_{m}}=\frac{\partial F_{n+m-1}}{\partial p}, \quad p_{t_{m}}=-\frac{\partial F_{n+m-1}}{\partial q}
$$

Proposition 3.2. The Hamiltonian systems in the last equation are completely integrable in the sense of Liouville, and if $(p, q)$ satisfies (2.25) and (3.11), then $v$ given by (2.18) and (2.19) is a solution of CHD equation.

Proof. Since $F_{k}$ are in involution, the systems $(3.11)(m=1,2, \ldots)$ are completely integrable. Observe that (2.3) is deduced from the solvability condition of (2.22) and (3.9); (2.25) and (3.11) are obtained by substituting (2.18) and (2.19) into (2.22) and (3.9), respectively. It is easy to see that if $(q, p)$ satisfies both (2.25) and (3.11), then $v$ given by (2.18) and (2.19) is a solution of CHD equation.

\section{References}

[1] M. Antonowicz and A. P. Fordy, Coupled Harry Dym equations with multi-Hamiltonian structures, Journal of Physics. A. Mathematical and General 21 (1988), no. 5, L269-L275.

[2] V. I. Arnold, Mathematical Methods of Classical Mechanics, Graduate Texts in Mathematics, vol. 60, Springer, New York, 1978.

[3] C. Cao, Confocal involutive systems and a class of AKNS eigenvalue problems, Henan Science 1 (1987), 1-10.

[4] _ Nonlinearization of the Lax system for AKNS hierarchy, Chinese Science. Series A 7 (1989), 701-707.

[5] _ A classical integrable system and the involutive representation of solutions of the KdV equation, Acta Mathematica Sinica. New Series 7 (1991), no. 3, 216-223.

[6] C. Gu (ed.), Soliton Theory and Its Applications, Springer, Berlin; Zhejiang Science and Technology Publishing House, Hangzhou, 1995.

[7] J. Moser, Integrable Hamiltonian systems and spectral theory, Proceedings of the 1983 Beijing Symposium on Differential Geometry and Differential Equations (L. Shantao, ed.), Science Press, Beijing, 1986, pp. 157-229.

[8] Z. J. Qiao, A Bargmann system and the involutive representation of solutions of the Levi hierarchy, Journal of Physics. A. Mathematical and General 26 (1993), no. 17, 4407-4417.

[9] _ The Camassa-Holm hierarchy, $N$-dimensional integrable systems, and algebro-geometric solution on a symplectic submanifold, Communications in Mathematical Physics 239 (2003), no. 1-2, 309-341. 
[10] T. Xu, A hierarchy of completely integrable Neumann systems associated with $y_{x x}=\left(u_{0}+u_{1} \lambda+\right.$ $\left.u_{2} \lambda^{2}+u_{3} \lambda^{3}-\lambda^{4}\right) y$, Northeastern Mathematical Journal 8 (1992), no. 1, 96-102.

[11] T. Xu and X. G. Geng, A completely integrable Neumann system in Liouville sense, Chinese Science Bulletin 35 (1990), no. 22, 1859-1861.

[12] T. Xu and Z. Gu, Lax representation for the higher-order Heisenberg equation, Chinese Science Bulletin 35 (1989), 1404-1406.

[13] T. Xu and W. Mu, Finite-dimensional completely integrable systems associated with the Harry Dym and the coupled Harry Dym hierarchies, Physics Letters. A 147 (1990), no. 2-3, 125-129.

[14] Y. B. Zeng, T. Xu, and Y. S. Li, A hierarchy of integrable Hamiltonian systems associated with $\phi_{x x}=\left(\lambda^{3}-u_{0}-\lambda u_{1}-\lambda^{2} u_{2}\right) \phi$, Physics Letters. A 144 (1990), no. 2, 75-80.

[15] Z. X. Zhou and W.-X. Ma, Finite dimensional integrable Hamiltonian systems associated with DSI equation by Bargmann constraints, Journal of the Physical Society of Japan 70 (2001), no. 5, $1241-1245$.

Taixi Xu: Department of Mathematics, Southern Polytechnic State University, 1100 South Marietta Parkway, Marietta, GA 30060, USA

E-mail address: txu@spsu.edu

Weihua Mu: Department of Mathematics, Shijiazhuang Railway Institute, Hebei 050043, China E-mail address: slx@sjzri.edu.cn

Zhijun Qiao: Department of Mathematics, University of Texas - Pan American, 1201 W. University Drive Edinburg, TX 78541, USA

E-mail address: qiao@utpa.edu 


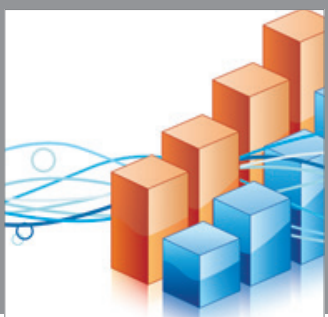

Advances in

Operations Research

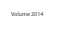

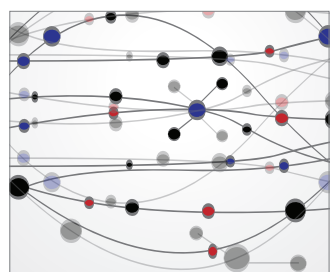

\section{The Scientific} World Journal
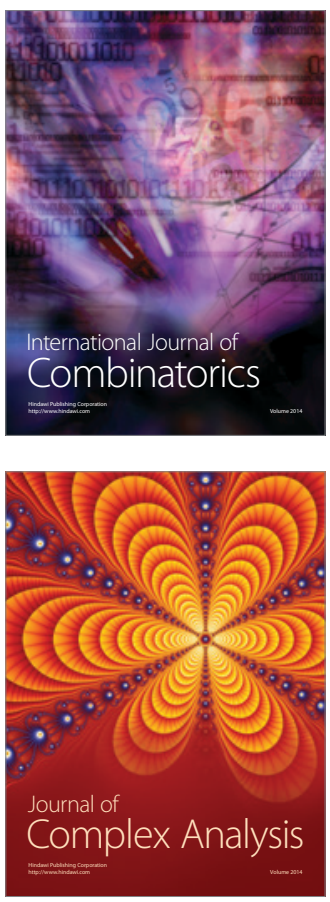

International Journal of

Mathematics and

Mathematical

Sciences
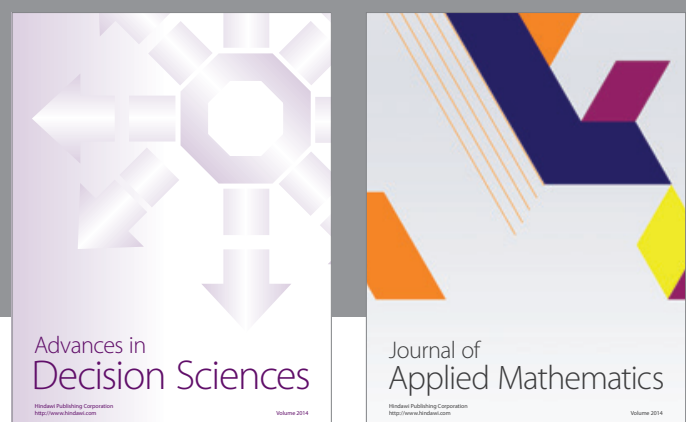

Journal of

Applied Mathematics
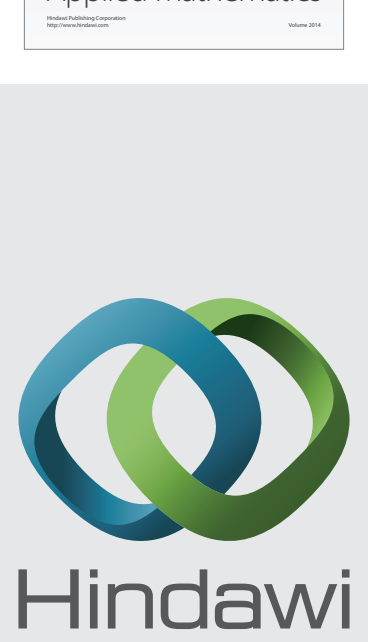

Submit your manuscripts at http://www.hindawi.com
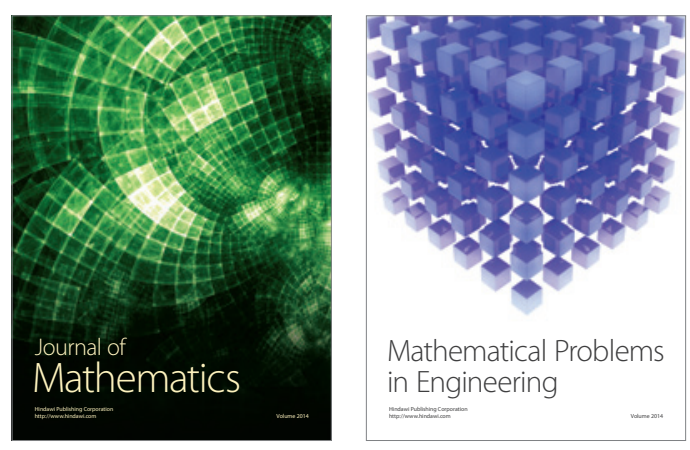

Mathematical Problems in Engineering
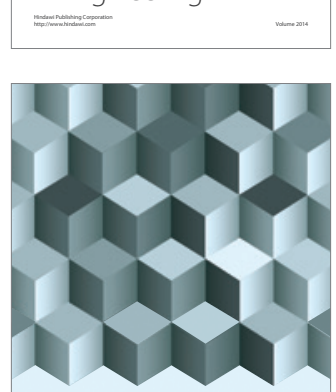

Journal of

Function Spaces
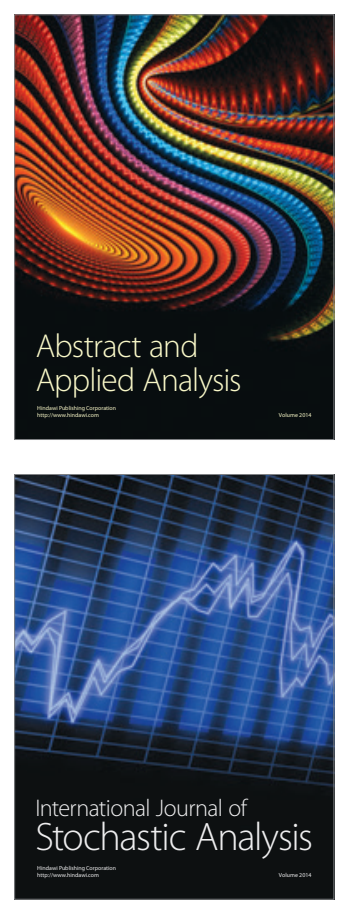

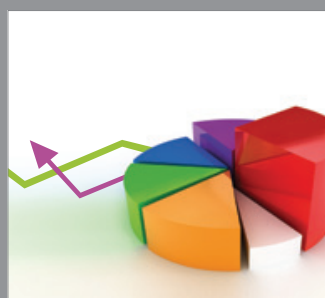

ournal of

Probability and Statistics

Promensencen
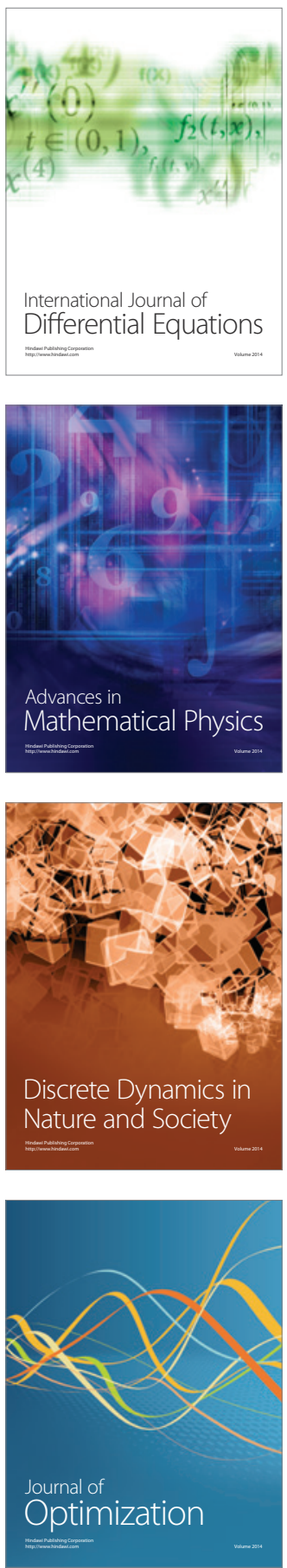